\title{
Induction of gastric epithelial apoptosis by Helicobacter pylori
}

\author{
S F Moss, J Calam, B Agarwal, S Wang, P R Holt
}

\begin{abstract}
Background-Helicobacter pylori may promote gastric carcinogenesis through increasing gastric epithelial cell proliferation. How $H$ pylori does so is unknown. Programmed, non-necrotic, cell death (apoptosis) occurs throughout the gut and is linked to proliferation. It was hypothesised that $H$ pylori may induce hyperproliferation through increasing apoptosis. Aim-To measure the effect of $H$ pylori infection on gastric epithelial apoptosis in situ.
\end{abstract}

Patients-Patients with duodenal ulcers treated to eradicate $H$ pylori and patients with $H$ pylori negative non-ulcer dyspepsia.

Methods-Retrospective quantification of apoptotic epithelial cells in situ from formalin fixed biopsy specimens, counted after staining by terminal uridine deoxynucleotidyl nick end-labelling.

Results-In the uninfected stomach, apoptotic cells were rare and situated in the most superficial portion of gastric glands (mean $2 \cdot 9 \%$ of epithelial cells). In $H$ pylori infection, they were more numerous and were located throughout the depth of gastric glands, comprising $16 \cdot 8 \%$ of epithelial cells, falling to $3 \cdot 1 \%$ after $H$ pylori eradication, $\mathbf{p}=0.017$. Apoptotic cell number did not correlate with the degree of histological gastritis.

Conclusions-These results suggest that $H$ pylori induces epithelial apoptosis in vivo. Increased apoptosis may be the stimulus for a compensatory hyperproliferative and potentially preneoplastic response in chronic $H$ pylori infection. (Gut 1996; 38: 498-501)

Department of Medicine, St Luke'sRoosevelt Hospital Center/Columbia University, NY, USA $S$ F Moss

B Agarwal

S Wang

P R Holt

Department of

Medicine,

Hammersmith

Hospital, London

J Calam

Correspondence to: Dr S F Moss, Division of Gastroenterology, Department of Medicine, S\&R 12, 1111 Amsterdam Ave, New York, NY 10025, USA.

Accepted for publication 23 October 1995
Keywords: Helicobacter pylori, apoptosis, gastric carcinogenesis.

Gastric Helicobacter pylori infection is associated with peptic ulceration, gastric lymphoma, and gastric carcinoma. ${ }^{1}$ How this organism interacts with the gastric epithelium to cause these diseases is still unclear. ${ }^{2}$ Although $H$ pylori has recently been defined as a definite carcinogen, ${ }^{3}$ how it might promote neoplasia is largely speculative. Proposed mechanisms for the carcinogenic effect of $H$ pylori infection include reduction of the gastric antioxidant ascorbate by $H$ pylori ${ }^{4}$ and stimulation of epithelial proliferation ${ }^{5}{ }^{6}$ because increased cell turnover predisposes to mutagenesis in the multistep model of gastric carcinogenesis. ${ }^{7} 8$

Mucosal epithelial hyperproliferation may be a response to $H$ pylori induced cell damage or death, yet there is a notable lack of epithelial necrosis in chronic $H$ pylori infection. ${ }^{9}$ Recently, however, it has been appreciated that necrosis is not the only form of cell death and that programmed cell death (apoptosis) is the physiological way in which cells die. ${ }^{1011}$ Apoptosis, in contrast with necrosis, occurs in single cells, in response to the expression of specific cellular genes ${ }^{12}$ and may therefore be considered a type of cellular suicide. Apoptosis is an essential part of the cycle of cellular turnover in many tissues, including the gastrointestinal tract. Tissue integrity is maintained when the rate of cell loss by apoptosis is matched by the rate of new cell production by proliferation. Furthermore, changes in the rate of apoptotic cell loss may contribute to diseases characterised by abnormalities of tissue growth. ${ }^{12}$ In $H$ pylori gastritis where there is increased epithelial proliferation and little or no necrotic cell death, we hypothesised that the increased cell proliferation in $H$ pylori gastritis must be accompanied by increased programmed (apoptotic) cell death

Apoptotic cells are very difficult to see by conventional light microscopy, especially in the presence of inflammation. One of the hallmarks of apoptosis, however, is the stepwise degradation of DNA by endonucleases, resulting in short fragments of double stranded DNA, enabling apoptotic cells to be identified in situ by incorporating labelled nucleotides onto the $3^{\prime}$ free hydroxyl ends by terminal deoxynucleotidyl transferase. ${ }^{10}{ }^{13}$ We therefore examined apoptosis in situ using the method of terminal uridine deoxynucleotide neck end labelling (TUNEL) in the 'normal' uninfected stomach and in the $H$ pylori infected stomach of duodenal ulcer patients, in whom we could examine the effect of $H$ pylori because biopsy specimens had been taken from these patients both before and after $H$ pylori eradication therapy. Our findings show that $H$ pylori infection is associated with increased epithelial apoptosis, suggesting that this is the stimulus that induces hyperproliferation and mutagenesis.

\section{Methods}

This study was a retrospective examination of formalin fixed paraffin wax embedded blocks of gastric antral biopsy specimens that had been collected for routine histopathology. Suitable cases were identified from the endoscopy clinic and medical records of Hammersmith Hospital, London and St Luke's/Roosevelt Hospital Center in New York City. We specifically excluded patients 
who had undergone previous gastric surgery or were known to be taking non-steroidal antiinflammatory drugs, corticosteroids or prostaglandin analogues, which may damage the gastric epithelium or impair the normal gastric cytoprotective response to injury. Patients were also excluded if they had taken antibiotics or other anti- $H$ pylori medication such as a bismuth compound or a proton pump inhibitor in the previous one month as these agents may have suppressed the numbers of $H$ pylori organisms. The study was approved by the research ethics committee of each hospital.

\section{Patients}

Controls - 12 patients who had undergone diagnostic endoscopy and biopsy in whom the final clinical diagnosis was non-ulcer dyspepsia and who were not infected with $H$ pylori. Seven were male, mean age 50 years, range 22 to 80 .

Duodenal ulcer patients - 16 patients with active duodenal ulceration and $H$ pylori associated chronic superficial gastritis were treated with colloidal bismuth subcitrate, tetracycline, and metronidazole. They had a repeat endoscopy and biopsy to confirm ulcer healing and eradication one month after the end of treatment, as described previously. ${ }^{14}$ Ten were male, mean age 53 years, range 21 to 83 .

\section{Diagnosis of $\mathrm{H}$ pylori infection}

At the time of endoscopy three antral biopsy specimens were taken $2 \mathrm{~cm}$ from the pylorus along the greater curve for urease test, histological examination, and culture as previously described. ${ }^{14}$ If any test was positive, the patient was considered to be infected.

\section{TUNEL histochemistry}

TUNEL histochemistry was performed in our laboratory using a procedure modified from that described by Gavrieli et al. ${ }^{10}$ Four $\mu \mathrm{m}$ sections were cut from formalin fixed, paraffin wax embedded tissue blocks and mounted on 'Superfrost-Plus' coated microscope slides (Fisher Scientific, Pittsburgh, PA). The sections were deparaffinised through xylene and alcohol, washed, and then digested with proteinase K $20 \mu \mathrm{g} / \mathrm{ml}$ (Sigma, St Louis, MO) for 15 minutes at room temperature, followed by further washing and blocking of endogenous peroxidase with $2 \%$ hydrogen peroxide (Sigma) for five minutes. After preincubation with terminal transferase buffer containing $200 \mathrm{mM}$ potassium cacodalyte, $0.2 \mathrm{mM}$ EDTA, $25 \mathrm{mM}$ TRIS-HCl, bovine serum albumin $0.25 \mathrm{mg} / \mathrm{ml}$, pH 6.6 (Boehringer Mannheim, Indianapolis, $\mathrm{IN}$ ) for 10 minutes, the sections were incubated at $37^{\circ} \mathrm{C}$ for 90 minutes with the same buffer containing, in addition, $1 \mathrm{mM}$ cobalt chloride, terminal transferase $(0.5 \mathrm{U} / \mu \mathrm{l})$, and $0.4 \mu \mathrm{M}$ digoxigenin-11-deoxyuridine triphosphate (dUTP), all purchased from Boehringer. The reaction was terminated with a solution of $300 \mathrm{mM}$ sodium chloride, $30 \mathrm{mM}$ sodium citrate and the slides were washed in water, followed by
$2 \%$ bovine serum albumin and then phosphate buffered saline. Detection of incorporated digoxigenin-11-dUTP was achieved by incubation with peroxidase conjugated $\mathrm{Fab}$ fragments of anti-digoxigenin (Boehringer) at a concentration of $1: 300$ in $100 \mathrm{mM}$ TRIS- $\mathrm{HCl}$, $150 \mathrm{mM}$ sodium chloride, $\mathrm{pH} 7.5$ at room temperature for 30 minutes. The slides were then washed in water and then $0.1 \mathrm{M}$ acetate buffer, $\mathrm{pH} 6$, and exposed to freshly prepared $1 \%$ diaminobenzene (Sigma) with nickel enhancement ${ }^{15}$ for 20 minutes, followed by counterstaining with methyl green, dehydration, and mounting. As a positive control, sections pretreated with DNAse in vitro at concentrations of $10 \mu \mathrm{g} / \mathrm{ml}$ to $1 \mathrm{mg} / \mathrm{ml}$ resulted in positive staining in all cells in a dose dependent manner as previously described. ${ }^{10}$ For negative controls either terminal deoxynucleotidyl transferase or digoxigenin-dUTP were omitted, resulting in uniformly negative staining. At least 300 epithelial cells were counted in each section and the number of positive cells per 100 cells was expressed as the apoptotic index (\%). Counting was by a single observer who was unaware of the clinical status of the patients. The interassay coefficient of variation in apoptotic index in our laboratory was $42 \%$ and the intra-assay variation $15 \%$.

\section{Assessment of gastritis}

In each case an adjacent tissue section was stained by haematoxylin and eosin and the degree of histological gastritis was scored from a minimum of zero to a maximum of 10 using the scoring system developed by Rauws et al ${ }^{16}$ as used previously. ${ }^{14}$

\section{Statistical analysis}

The Mann-Whitney U test was used to compare scores between clinicopathological groups. Differences between apoptotic indices in the same patients before and after eradication of $H$ pylori were compared by the Wilcoxon signed rank test.

\section{Results}

\section{Gastric apoptosis}

Apoptotic bodies were very rarely identified in the gastric epithelium in haematoxylin and eosin stained sections at $400 \times$ magnification. In contrast, cells that had stained positively by TUNEL could be seen even at $100 \times$ magnification. In the 'normal' stomach of non-ulcer dyspepsia patients without $H$ pylori infection, apoptotic epithelial cells were infrequently identified by TUNEL histochemistry. Positively stained cells were located in the most superficial part of the gastric gland, comprising a mean of $2.9 \%$ (median 2 , range $0-9$ ) of all gastric epithelial cells (Fig 1). In duodenal ulcer patients infected with $H$ pylori, apoptotic epithelial cells were more numerous and they were also seen deep in the glands (Fig 2). In addition, positively stained cells could be identified within the lamina propria. The mean 


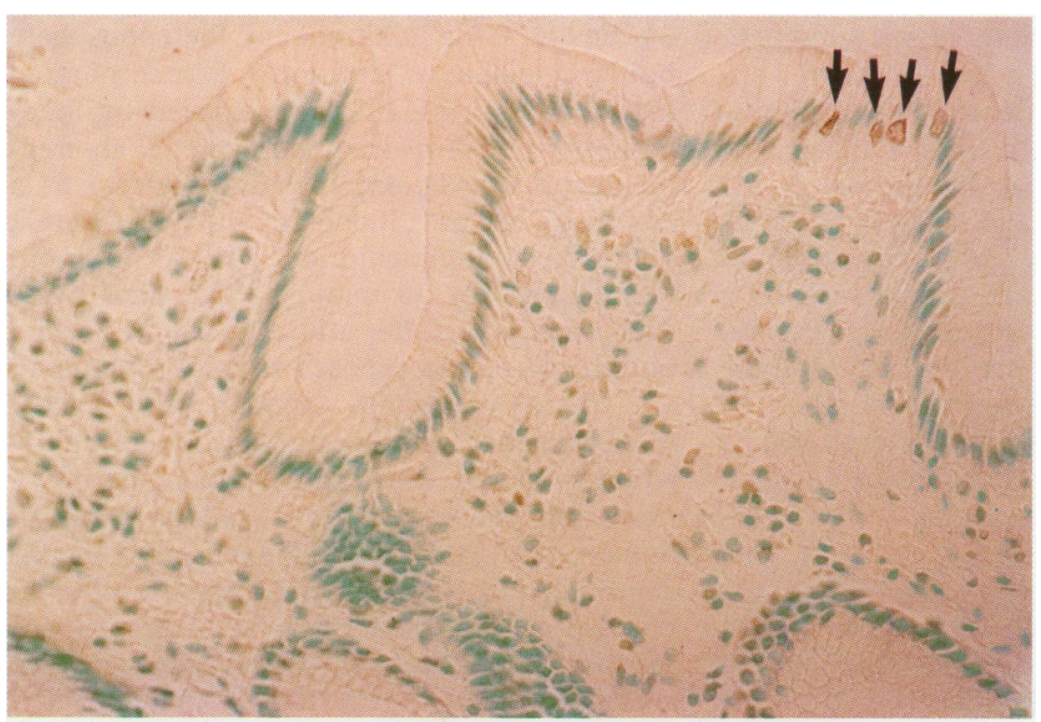

Figure 1: Photomicrograph of gastric antral biopsy specimen from a $\mathrm{H}$ pylori negative patient with non-ulcer dyspepsia. Occasional apoptotic cells are present in the most superficial area of the epithelium (arrows). TUNEL stain. Original magnification $\times 400$.

epithelial apoptotic index was $14 \cdot 1 \%$ (median $6 \cdot 5$, range $0-44), p=0.048$ versus controls. After triple therapy, $H$ pylor $i$ was cured in 12 of 16 patients and in these patients the mean apoptotic index fell from $16 \cdot 8 \%$ (median $7 \cdot 5$, range $0-44$ ) to $3 \cdot 1 \%$ (median $1 \cdot 5$, range $0-18$ ), $\mathrm{p}=0 \cdot 017$, Wilcoxon signed rank test (Fig 3 ). In the four patients in whom $H$ pylori was not cured, the apoptotic index fell from a mean of $6.8 \%$ (median 4.5 , range $0-18$ ) to $0.9 \%$ (median $1 \cdot 0$, range $0-1 \cdot 5$ ).

\section{Lack of correlation between apoptosis and inflammation}

The fall in apoptotic index in the duodenal ulcer patients may result from the associated improvement in gastritis when $H$ pylori is eradicated, particularly in view of the rapid resolution of neutrophil infiltration accompanying $H$ pylori eradication. ${ }^{9}$ We therefore examined the relation between apoptotic index and either neutrophil or total gastritis scores. In non-ulcer dyspeptic controls the mean total gastritis score was 0.36 (median 0 , range $0-1$ )

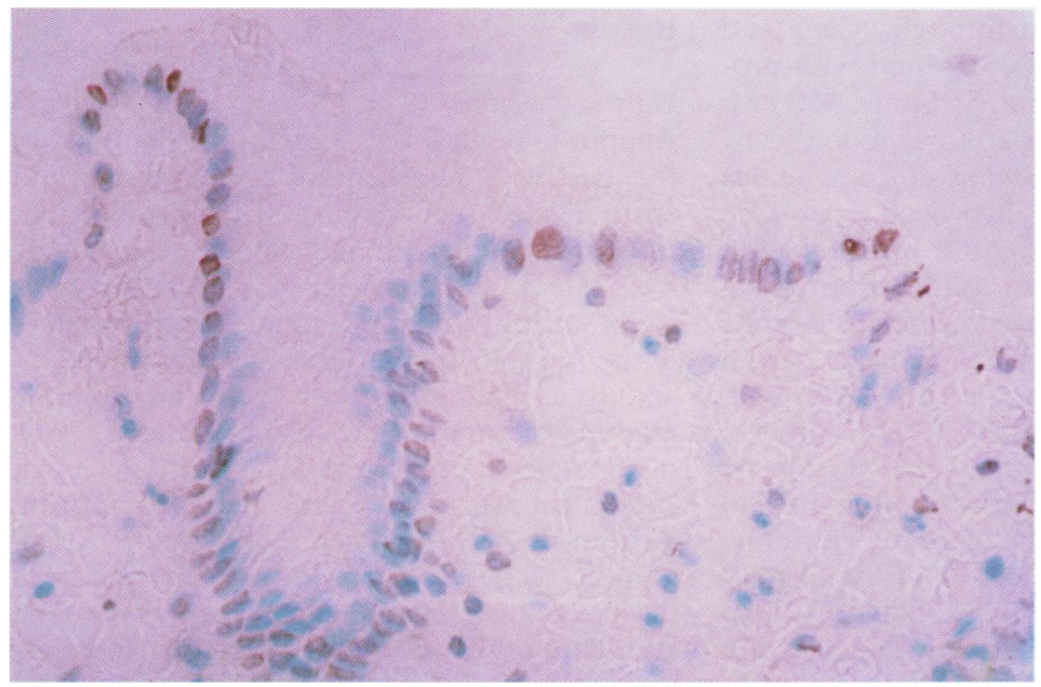

Figure 2: Numerous brown stained nuclei of apoptotic epithelial cells in the antral biopsy specimen from a duodenal ulcer patient infected by $\mathrm{H}$ pylori. TUNEL stain. Original magnification $\times 400$. and the neutrophil score was 0 in all cases (Table). On eradication of $H$ pylori in the duodenal ulcer patients, the mean gastritis score decreased from $3 \cdot 7$ (median $3 \cdot 5$, range $1-8$ ) to 1.9 (median 1 , range $1-6$ ), $p=0.024$, and the neutrophil gastritis score fell from 1.5 (median 1 , range $0-5$ ) to 0.5 (median 0 , range $0-2$ ), $\mathrm{p}=0.023$. In contrast, in the four patients in whom $H$ pylori was not cured the mean total and neutrophil gastritis scores were 3.8 and 1.25 before and 3.5 and 0.8 after treatment respectively. The gastritis scores were in all cases significantly higher in the duodenal ulcer patient than in the controls, even after eradication ( $p<0.0001$ in all cases, Table). Overall, there was no correlation between the apoptotic index and either the neutrophil score or the total gastritis score $(p=0.22$ and $p=0.20$ respectively).

\section{Discussion}

These results show that $H$ pylori infection is associated with increased epithelial apoptosis and that after treatment to eradicate $H$ pylori, apoptosis decreases to that found in normal controls. This implies that $H$ pylori induces gastric epithelial apoptosis and may explain how $H$ pylori promotes a hyperproliferative response. Although the increased proliferation may be considered an appropriate physiological response to maintain tissue mass, if hyperproliferation persists it may eventually produce cells that are more resistant to apoptosis, thereby leading to tissue growth and neoplasia, as has been found in colonic carcinogenesis. ${ }^{17} 18$ In support of this hypothesis, Lauwers et al found that the expression of the anti-apoptotic protein bcl-2 was increased in precancerous gastric lesions, particularly in gastric epithelial dysplasia. ${ }^{19}$

How $H$ pylori promotes apoptosis is not known. In attempting to understand the pathophysiological mechanisms of $H$ pylori it is important to separate the direct effect of $H$ pylori from the effect of the accompanying inflammatory infiltrate. Our results favour a direct bacterial effect as there was no correlation between the extent of inflammation, assessed histologically, and the apoptotic index. In addition, the control group had similar apoptotic indices to the duodenal ulcer patients in whom $H$ pylori had been cured although the duodenal ulcer patients had persistent inflammatory cells one month after the end of treatment. Furthermore, there was a reduction in apoptotic index in the few duodenal ulcer patients in whom $H$ pylori was eradicated although there was no change in inflammatory score. We suspect that the fall in apoptotic index in these patients may result from a reduction in bacterial load after unsuccessful $H$ pylori eradication therapy but cannot exclude the possibility that a component of the triple therapy may have been directly responsible for reducing the epithelial apoptotic index to normal, regardless of its effect on $H$ pylori.

Interestingly, the four patients with the highest apoptotic indices before treatment had no evidence of atrophy or intestinal metaplasia on antral biopsy, whereas seven of eight 


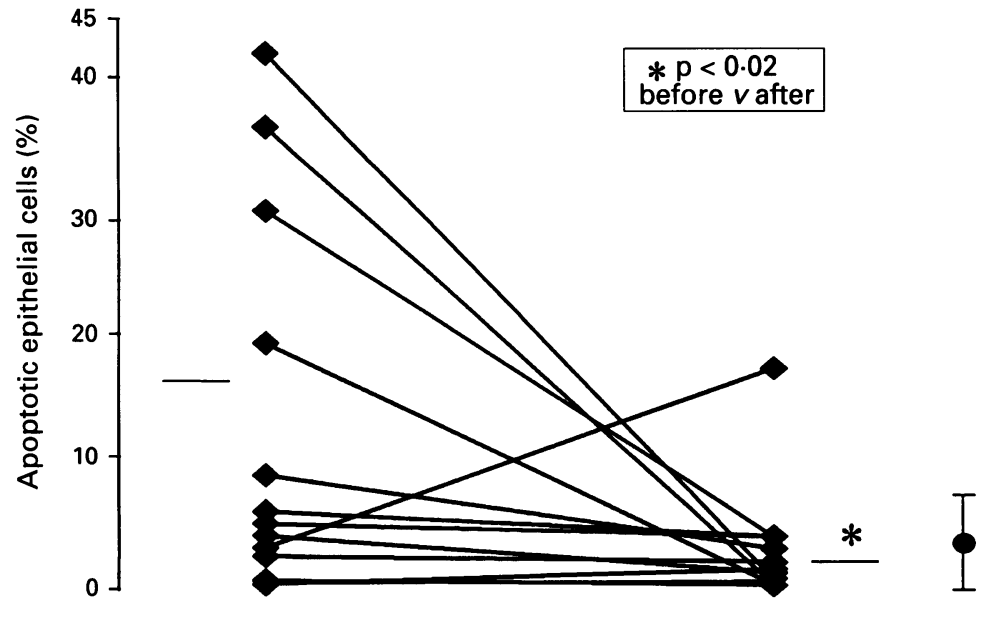

Before treatment

After treatment $\mathrm{HP}^{-}$NUD

Figure 3: The effect of eradication of $\mathrm{H}$ pylori on gastric epithelial apoptosis. The data points represent 12 patients in whom $\mathrm{H}$ pylori was successfully eradicated, with mean values shown by horizontal bars. The $\mathrm{H}$ pylori negative non-ulcer dyspepsia (HP- NUD) group of controls are shown on the right (mean (SD)).

patients with pretreatment apoptotic scores less than $20 \%$ did. This may suggest that the induction of apoptosis occurs comparatively early in the natural history of $H$ pylori infection, followed later by a compensatory decrease in apoptotic activity during the progression of atrophic gastritis and intestinal metaplasia.

Precisely how $H$ pylori infection induces apoptosis is not apparent from our data. Several bacterial products may be directly responsible for the induction of apoptosis that we observed, including the CagA and VacA proteins. As our patients had duodenal ulcer they were probably infected with $H$ pylori strains expressing these products, ${ }^{2}$ but whether all strains promote apoptosis remains to be investigated. Another candidate bacterial virulence factor is ammonia, generated by the breakdown of urea by $H$ pylori's urease, as ammonia has been shown to induce gastric epithelial apoptosis in vitro in rat cell lines. ${ }^{20}$ Whether the promotion of apoptosis is caused

Gastritis scores

\begin{tabular}{llll}
\hline & Number & Total gastritis score & $\begin{array}{l}\text { Neutrophil } \\
\text { gastritis score }\end{array}$ \\
\hline Controls $(H$ pylori negative) & 12 & $0 \cdot 4(0 \cdot 5)$ & $0(0)$ \\
H pylori positive before treatment & 16 & $3 \cdot 7(1 \cdot 9)^{\star}$ & $1 \cdot 4(1 \cdot 5) \ddagger$ \\
H pylori positive after successful treatment & 12 & $1.9(1.5) \dagger \ddagger$ & $0.5(0.7) \neq \emptyset$ \\
\hline
\end{tabular}

Gastritis scores are expressed as mean (SD). Gastritis was assessed histologically. ${ }^{16}$

${ }^{\star} p<0.0001$ versus controls. $\neq p=0.02$ versus before treatment. $\neq p<0.001$ versus controls. $\mathrm{p}=0.02$ versus controls. by the bacteria directly or by inflammatory infiltrate, the induction of apoptosis by $\mathrm{H}$ pylori provides an explanation linking chronic infection by this organism with a compensatory hyperproliferative and potentially neoplastic response.

This work was presented in abstract form at Digestive Disease Week, San Diego, May 1995.

$1 \mathrm{NIH}$ Consensus Conference. Helicobacter pylori in peptic ulcer disease. NIH Consensus development panel. $\mathfrak{f} A M A$ 1994; 272: 65-9.

2 Blaser MJ, Parsonnet J. Parasitism by the 'slow' gastric bacterium Helicobacter pylori leads to altered gastric homeostasis and neoplasia. $\mathcal{f}$ Clin Invest 1994; 94: 4-8.

3 Anonymous. Schistosomes, liver flukes and Helicobacter pylori. IARC Working group on the evaluation of carcinogenic risks to humans. Lyon: 1994. IARC monographs on the evaluation of carcinogenic risks to humans. 1994; 61: $1-241$

4 Sobala GM, Schorah CJ, Shires S, Lynch DA, Gallacher B, Dixon MF, et al. Effect of eradication of Helicobacter pylori on gastric juice ascorbic acid concentrations. Gut 1993; 34: 1038-41.

5 Brenes F, Ruiz B, Correa P, Hunter F, Rhamakrishnan T, Fontham E, et al. Helicobacter pylori causes hyperproliferation of the gastric epithelium: pre-and post-eradication
indices of proliferating cell nuclear antigen. $A m ~$ indices of proliferating cell

6 Lynch DAF, Mapstone NP, Clarke AMT, Sobala GM, Jackson P, Morrison L, et al. Cell proliferation in Helicobacter pylori associated gastritis and the effect of eradication therapy. Gut 1995; 36: 346-50.

7 Correa P, Shiao Y-H. Phenotypic and genotypic events in gastric carcinogenesis. Cancer Res 1994; 54 (suppl 7): 1941-3s.

8 Ames BN, Gold LS. Chemical carcinogenesis: too many rodent carcinogens. Proc Natl Acad Sci USA 1990; 87: 7772-6.

9 Robert ME, Weinstein WM. Helicobacter pylori-associated gastric pathology. Gastroenterol Clin North Am 1993; 22: $59-72$.

10 Gavrieli Y, Sherman Y, Ben-Sasson SA. Identification of programmed cell death via specific labelling of nuclear DNA fragmentation. $\mathcal{f}$ Cell Biol 1992; 119: 493-501.

11 Hall PA, Coates PJ, Ansari A, Hopwood D. Regulation of cell number in the mammalian gastrointestinal tract: the importance of apoptosis. F Cell Sci 1994; 107: 3569-77.

12 Thompson CB. Apoptosis in the pathogenesis and treatment of disease. Science 1995; 267: 1456-62.

13 Coates PJ. Molecular methods for the detection of apoptosis in tissue. Fournal of Histotechnology 1994; 17: 261-7.

14 Moss SF, Legon S, Davies J, Calam J. Cytokine gene expression in Helicobacter pylori-associated antral gasexpression in Helicobacter pylo

15 Shu S, Ju G, Fan L. The glucose oxidase-DAB-nickel method in peroxidase histochemistry of the nervous method in peroxidase histochemistry
system. Neurosci Lett 1988; 85: 169-71.

16 Rauws EAJ, Langenberg W, Houthoff HJ, Zanen HC, Tytgat GNJ. Campylobacter pyloridis-associated chronic antral gastritis. A prospective study of its prevalence and the effects of antibacterial and antiulcer treatment. Gastroenterology 1988; 94: 33-40.

17 Bedi A, Pasricha PJ, Akhtar AJ, Barber JP, Bedi GC, Giardiello FM, et al. Inhibition of apoptosis during development of colorectal cancer. Cancer Res 1995; 55: 1811-6.

18 Moss SF, Scholes J, Wang S, Holt PR. Progressively disordered apoptosis during the multistep process of colorectal carcinogenesis. Gastroenterology 1995; 108: A511.

19 Lauwers GY, Scott GV, Hendricks J. Immunohistochemical evidence of aberrant bcl-2 protein expression in gastric epithelial dysplasia. Cancer 1994; 73: 2900-4.

20 Tsuji S, Kawano S, Takei Y, Tsuji M, Koabyashi I, Nagano $\mathrm{K}$, et al. Ammonia induces gastric cell apoptosis: possible implication to Helicobacter-related gastric mucosal atrophy. Gastroenterology 1995; 108: A244. 